\title{
26. Tätigkeitsbericht 2018/2019
}

\section{Die Schweiz muss Datenschutzniveau halten}

Der EDÖB erwartet mit der Vorlage des 26. Tätigkeitsberichts am 18. Juni 2019, dass Bundesrat und Parlament der Schweizer Bevölkerung durch eine baldige Unterzeichnung der Europaratskonvention 108 und den zügigen Abschluss der Totalrevision des Datenschutzgesetzes weiterhin ein mit dem europäischen Umfeld abgestimmtes Schutzniveau gewährleisten. Bei seiner Datenschutzaufsicht setzt der Beauftragte Schwerpunkte bei den Sicherheitsbehörden des Bundes und der Firma SwissSign. Bezüglich Öffentlichkeitsgesetz setzt sich die schon im Vorjahr festgestellte Konsolidierung fort.

\section{Forderung nach einer baldigen Beendigung der schwierigen Übergangszeit}

Die Totalrevision des Bundesgesetzes über den Datenschutz (DSG) ist mit der Botschaft des Bundesrats im September 2017 in den legislativen Prozess eingetreten, ohne dass das Gesetz bis dato in einer Kammer beraten worden ist. Seit einem Jahr indes gilt im europäischen Umfeld einheitlich die neue Datenschutzgrundverordnung (DSGVO). Die andauernde Übergangszeit bis zum Inkrafttreten des totalrevidierten Gesetzes gestaltet sich indessen nach wie vor als herausfordernd. Während die personell verstärkten Datenschutzbehörden in den Staaten des EWR inzwischen ihre neuen Verfügungs- und Sanktionsbefugnisse zum Tragen bringen, verfügt der EDÖB gegenüber der Wirtschaft und dem Gros der Bundesbehörden bis auf Weiteres nur über die im DSG von 1992 vorgesehenen Empfehlungsbefugnisse. Auch seine Mittel sind seit dem Jahre $2005 \mathrm{im}$ Wesentlichen unverändert geblieben, was dazu führte, dass die Europäische Kommission im März 2019 die Kontrolldichte und Mittelausstattung der Eidgenössischen Datenschutzaufsicht im Zuge ihrer Schengen-Evaluation als ungenügend beurteilte.

\section{Schengen-DSG verstärkt Aufsicht über die Sicherheitsbehörden des Bundes}

Mit dem am 1. März 2019 in Kraft getretenen Schengen-Datenschutzgesetz (SDSG), das die Personendatenbearbeitung im Polizeibereich regelt, wurde der EDÖB mit zusätzlichen Aufgaben und Befugnissen betraut. Der Beauftragte erwartet nun, dass ihm der Bundesrat Personalressourcen zur Verfügung stellen wird, damit er bei der Aufsicht der Sicherheitsbehörden des Bundes einen entsprechenden Schwerpunkt setzen kann.

In diesem Zusammenhang beobachtet der EDÖB auch den weltweiten Trend der Sicherheitsbehörden zur intensivierten Bearbeitung von biometrischen Daten durch Einsatz von Technologien wie DNA-Profiling, Gesichtserkennung oder Voiceprint. Mit Blick auf diese Intensivierung fordert er hinreichend bestimmte und verhältnismäßige Rechtsgrundlagen.
Kein gutes Beispiel gibt in dieser Hinsicht der Entwurf zum Bundesgesetz über polizeiliche Maßnahmen zur Bekämpfung von Terrorismus (PMT) ab. Mit unzureichend konkretisierten Datenbearbeitungen wird dieser aus Sicht des Beauftragten verunglückte Spezialerlass das bestehende Wirrwarr an spezialgesetzlichen Vorschriften auf Stufe des Bundespolizeirechts noch weiter verschärfen.

\section{E-ID und SwissSign}

Anlässlich der Anhörung in den Rechtskommissionen beider Räte sah der EDÖB seine Auf-gabe darin, sich unabhängig vom politischen Entscheid zugunsten einer rein staatlichen oder nur teilstaatlichen Lösung, für ein höchstmögliches Niveau an Datenschutz einzusetzen. Der Beauftragte hat denn auch diverse Verbesserungen des E-ID-Gesetzes verlangt. So haben National- und Ständerat als Anerkennungsvoraussetzung die vorgängige Anhörung des EDÖB durch die Zulassungsbehörde in das Gesetz aufgenommen. Desweitern haben wir darauf bestanden, dass die Botschaft dahingehend präzisiert wird, dass die E-ID nur dort Anwendung finden soll, wo eine sichere Identifikation im Geschäftsverkehr unbedingt nötig ist. Für die zahlreichen Online-Konsumgeschäfte oder Bezüge von einfachen Dienstleistungen, wo dies hingegen nicht nötig ist, dürfen durch das E-ID-Gesetz weder im analogen noch im elektronischen Geschäftsverkehr neue Identifizierungspflichten geschaffen werden. Die beiden Parlamentskammern haben den Zweckartikel des Gesetzes entsprechend angepasst.

Mit Blick auf die Beaufsichtigung der mit erheblichen Datenschutzrisiken verbundenen Tätigkeit der Firma SwissSign wird der EDÖB darauf hinwirken, dass der inzwischen eingesetzte betriebliche Datenschutz, die mit dem Betrieb von Single Sign In-Lösungen verbundenen Risiken transparent aufzeigt und dass die Firma diesen Risiken gerade auch mit Blick auf die von ihr angestrebte Zulassung als Providerin nach dem E-ID Gesetz mit vorbildlichen Schutzmaßnahmen und Investitionen in datenschutzfreundliche Technologien entgegen-wirkt.

\section{Die Bundesverwaltung zeigt sich transparenter}

Der EDÖB stellt fest, dass sich die Anwendung des Öffentlichkeitsgesetzes (BGÖ) durch die Bundesbehörden weiter konsolidiert.

Weiter stellt der Beauftragte generell eine aktivere Informationspolitik der Bundesverwaltung fest, die maßgeblich auf die Fortschritte zurückzuführen sein dürfte, die bei der Umsetzung des BGÖ erreicht werden konnten. Diese Entwicklung sowie der er-höhte Anteil einvernehmlicher Lösungen und weitere Effizienzsteigerungen im Schlichtungsverfahren vor dem EDÖB haben letztlich auch dazu geführt, dass der Bundesrat im Mai 2019 auf eine Revision des BGÖ verzichten konnte.

Der 26. Tätigkeitsbericht 2018/2019 des EDÖB kann auf $w w w$. edoeb.admin.ch als e-Paper heruntergeladen werden. 